\title{
Comparison of the Release Profile and Pharmacokinetics of Intact and Fragmented Dexamethasone Intravitreal Implants in Rabbit Eyes
}

\author{
Rahul Bhagat, ${ }^{1}$ Jean Zhang, ${ }^{2}$ Sidiq Farooq, ${ }^{2}$ and Xiao-Yan Li
}

\begin{abstract}
Purpose: Dexamethasone intravitreal implant (DEX implant, Ozurdex ${ }^{\circledR}$; Allergan, Inc.) is used to treat noninfectious posterior uveitis and macular edema associated with retinal vein occlusion and diabetic retinopathy. Two recently published reports of DEX implant fragmentation shortly after injection have raised concerns about the potential for faster implant dissolution and elevated ocular dexamethasone concentrations. This study compared the in vivo release profile and pharmacokinetic behavior of intact and fragmented DEX implants.

Methods: DEX implant was surgically implanted as a single unit or fragmented into 3 pieces in the posterior segment of opposing eyes of $36 \mathrm{New}$ Zealand white rabbits. The release of dexamethasone over time from 1-piece and 3-piece fragmented implants dissolved in solution in vitro was compared with that from the 1-piece and 3-piece fragmented implants placed in the rabbit eyes. In addition, dexamethasone concentrations in the vitreous and aqueous humors of each eye were measured at $3 \mathrm{~h}$ and days 1, 7, 14, 21, and 28. High-performance liquid chromatography and liquid chromatography-tandem mass spectrometry were used for assays.

Results: Dexamethasone release from the 1-piece and 3-piece DEX implants in vivo was not different and was consistent with the in vitro release pattern. Moreover, the concentration profile of dexamethasone in the vitreous and aqueous humors was similar for the 1-piece and 3-piece DEX implants at each time point measured.

Conclusions: DEX implant fragmentation neither accelerated its dissolution nor increased the dexamethasone concentration delivered at a given time. Accordingly, DEX implant fragmentation is unlikely to have clinically significant effects in patients.
\end{abstract}

\section{Introduction}

$\mathbf{R}$ ETINAL VEIN OCCLUSION (RVO), which includes branch RVO and central RVO, is the second most common retinal vascular disease worldwide after diabetic retinopathy. ${ }^{1,2}$ It is characterized by variable degrees of ischemia and edema, which frequently lead to vision loss and blindness if inadequately treated. ${ }^{1-3}$ Uveitis is also a leading cause of visual impairment, ${ }^{3}$ accounting for 30,000 new cases of legal blindness annually in the Western world. ${ }^{4}$ Posterior uveitis affects the retina, choroid, and occasionally the optic nerve and comprises $10 \%-40 \%$ of all uveitis cases. ${ }^{4}$ However, more cases of visual loss caused by inflammation and macular edema (among others) are associated with posterior uveitis than anterior or intermediate uveitis. ${ }^{4}$ Treating inflammation and edema is thus critical to improving outcomes in RVO and uveitis.
Corticosteroids such as dexamethasone, triamcinolone, and fluocinolone are the first-line therapy for noninfectious uveitis. ${ }^{3,4}$ They are also used to inhibit various proinflammatory mediators that contribute to RVO pathogenesis. ${ }^{1,3}$ Dexamethasone intravitreal implant (DEX implant, Ozurdex ${ }^{\circledR}$; Allergan, Inc., Irvine, CA $)^{5}$ was developed to provide sustained delivery of dexamethasone (which typically has a short half-life of 3-6h in solution), while retaining advantage of its potency (3-6 times higher compared with triamcinolone). ${ }^{4,6}$ DEX implant is made of a polylactic acid-glycolic acid matrix that is gradually converted into $\mathrm{CO}_{2}$ and water in vivo and eliminated by ocular tissues. ${ }^{4}$ As the matrix dissolves, impregnated dexamethasone is slowly released into the vitreous and retina where it alleviates edema and inflammation, ${ }^{4}$ starting as soon as 1 day after injection. ${ }^{7}$

\footnotetext{
${ }^{1}$ Allergan, Inc., Irvine, California.

${ }^{2}$ Oculex, Sunnyvale, California.
} 
Several large studies in patients with persistent macular edema caused by RVO, uveitis, diabetic retinopathy, or other causes showed that a single DEX implant $0.7 \mathrm{mg}$ significantly improves best-corrected visual acuity (BCVA). ${ }^{8-16}$ Notably, the sham-controlled randomized phase 3 GENEVA trials, including 1,267 patients with RVO-associated macular edema and vision loss, demonstrated that the DEX implant reduced the risk of vision loss $(P<0.001)$ and accelerated visual improvements $(P<0.001)$ without raising the incidence of cataracts or increased intraocular pressure (IOP), compared with controls. ${ }^{9}$ In the sham-controlled, phase 3 HURON trial, including 229 patients with noninfectious posterior uveitis, a single DEX implant $0.7 \mathrm{mg}$ improved intraocular inflammation and BCVA for over 6 months. ${ }^{10,11}$ The proportion of eyes with a vitreous haze score of 0 at 8 weeks was $47 \%$ in the DEX implant group versus $12 \%$ in the sham group $(P<0.001)$. Moreover, the incidence of cataracts and increased IOP was similar between groups $(P>0.05),{ }^{10}$ consistent with results from an earlier study. ${ }^{12}$ Thus, the $0.7-\mathrm{mg}$ dose has been approved by the Food and Drug Administration as first-line therapy for RVO and uveitis since 2009 and 2010, respectively, and is being used worldwide in 45 countries. ${ }^{3,13,14}$ The efficacy and safety of DEX implant was also recently demonstrated in diabetic macular edema, ${ }^{15}$ which led to its approval for this indication in adult patients.

Recently, 2 reports have been described in the literature in which the DEX implant has split into 2 pieces shortly after injection. ${ }^{16,17}$ Concerns regarding the potential for faster dissolution of the fragmented implant (compared with an intact implant) and higher drug concentrations were raised. ${ }^{18}$ We report herein results from an in vivo study in rabbits showing that the pharmacokinetic behavior of intact (1-piece) and fragmented (3-piece) DEX implants does not differ.

\section{Methods}

\section{Reagents}

Solvents were high-pressure liquid chromatography (HPLC) grade (EMD Millipore, Gibbstown, NJ). Deionized water was obtained from a Milli-Q system from Millipore Corporation (Billerica, MA). All other chemicals were analytical grade from Sigma-Aldrich (St. Louis, MO). DEX filament was obtained from Allergan, Inc.

\section{Preparation of DEX implants}

One-piece (lot No. 247-01) and 3-piece (lot No. 247-03) DEX implants were prepared from a single lot of filament (lot No. 238-01a). The 1-piece DEX implants were cut to an approximate weight of 1,050-1,284 $\mu \mathrm{g}$, and each fragment of the 3-piece DEX implants was cut to a weight of 350-428 $\mu \mathrm{g}$ [for a total of 1,050-1,284 $\mu \mathrm{g}$ after loading 3 fragments into a trocar (Covidien, Mansfield, MA, or equivalent)]. The average (range) dose of dexamethasone in the 1-piece and 3-piece DEX implants was $0.7 \mathrm{mg}(630-770 \mu \mathrm{g})$.

\section{In vitro assessment of dexamethasone release over time from 1-piece and 3-piece DEX implants}

Six 1-piece and 3-piece DEX implants were dissolved at $37^{\circ} \mathrm{C} \pm 1^{\circ} \mathrm{C}$ in $30 \mathrm{~mL}$ saline for $\geq 1 \mathrm{~h}$ until complete dissolution. At days $1(24 \pm 1 \mathrm{~h}), 4(96 \pm 1 \mathrm{~h}), 7(168 \pm 2 \mathrm{~h}), 14$
(336 $\pm 4 \mathrm{~h}), 21(504 \pm 4 \mathrm{~h})$, and $28(672 \pm 4 \mathrm{~h}), 25 \mathrm{~mL}$ was removed for HPLC analysis and replaced with $25 \mathrm{~mL}$ saline. Samples were then returned to $37^{\circ} \mathrm{C}$. Chromatographic separation of dexamethasone was achieved on a Waters Alliance HPLC system using a Rainin Varian Microsorb MV, C18, $5-\mu \mathrm{m}$ column $(4.6 \times 250 \mathrm{~mm})$ and a mobile phase of $44: 55$ acetonitrile/water. Detection was at $238 \mathrm{~nm}$ on a photodiode array detector. The percentage of dexamethasone released at each time point was calculated as follows, based on the label strength (LS) of $700 \mu \mathrm{g}$ dexamethasone per implant:

Day 1:\% $\mathrm{DEX}_{1}=$ Sample concentration $_{1} \times 30_{\mathrm{mL}} \times \frac{100 \%}{\mathrm{LS}}$

Day $4: \% \mathrm{DEX}_{4}=\left(\right.$ sample concentration $_{4} \times 30_{\mathrm{mL}}+$ sample concentration $\left._{1} \times 25_{\mathrm{mL}}\right) \times \frac{100 \%}{\mathrm{LS}}$

Day $7: \% \mathrm{DEX}_{7}=\left(\right.$ sample concentration $_{7} \times 30_{\mathrm{mL}}+$ sample concentration $_{4} \times 25_{\mathrm{mL}}+$ sample concentration $\left._{1} \times 25 \mathrm{~mL}\right) \times \frac{100 \%}{\mathrm{LS}}$, and so on for days 14,21 , and 28 .

\section{Surgical placement of DEX implant in rabbit eyes and sampling procedure}

All animal procedures were performed in accordance with the Association for Research in Vision and Ophthalmology Statement for the Use of Animals in Ophthalmic and Vision Research. The research was approved by an institutional review board and conducted in accordance with Good Laboratory Practices.

Thirty-six New Zealand white rabbits were implanted with a single DEX implant into the posterior segment of 1 eye and a fragmented 3-piece DEX implant into the posterior segment of the opposite eye as follows: animals were weighed and anesthetized with an intravenous injection of ketamine $87 \mathrm{mg} / \mathrm{mL}$-xylazine $13 \mathrm{mg} / \mathrm{mL}$ cocktail (SigmaAldrich, or equivalent) at $0.1-0.2 \mathrm{~mL} / \mathrm{kg}$. Ten minutes before surgery, all eyes were topically treated with 2 drops of Betadine $^{\circledR}$ 2.5\% solution (Purdue Pharma, Stamford, CT, or equivalent) for 2-5 min, washed with sterile saline, and topically treated with 1-2 drops of proparacaine hydrochloride $0.5 \%$ (AKORN, Inc., Lake Forest, IL, or equivalent). A sclerotomy was then performed on the right eye (between 10 and 12 o'clock) and on the left eye (between 1 and 2 o'clock), using a 20-gauge MVR blade (Rumex International, Clearwater, FL, or equivalent). Vitreous humor (50$100 \mu \mathrm{L}$ ) was removed by paracentesis, and a sterile trocar preloaded with a single-unit DEX implant or a 3-piece DEX implant was inserted $\sim 5 \mathrm{~mm}$ in the incision for injection. The incision was then closed with 7.0 Vicryl suture (Ethicon, Inc., Somerville, NJ, or equivalent), and each animal was monitored daily for symptoms of endophthalmitis (e.g., conjunctival congestion, swelling, and discharge).

At $3 \mathrm{~h}$ and days $1,7,14,21$, and 28 postimplantation, 6 animals were euthanized with an intravenous injection of commercial euthanasia solution (e.g., Euthasol ${ }^{\mathbb{B}}$; Virbac, 
Fort Worth, TX, or equivalent). Aqueous humor was then separately collected from both eyes, after which the globes were enucleated and frozen at $-70^{\circ} \mathrm{C}$ for at least $1 \mathrm{~h}$ to allow each vitreous humor to be divided into 2 (one-half with the implant remnants, the other remnant-free). Vitreous humor with and without DEX remnants and aqueous humor samples were stored at $-70^{\circ} \mathrm{C}$ until analysis.

\section{Determination of the percentage of dexamethasone released by HPLC analysis of vitreous humor containing DEX implant remnants}

The volume of each vitreous sample containing DEX implant remnants was measured before dissolving in $10 \%$ acetonitrile (2.5-5 mL), sonicating for $30 \mathrm{~min}$ in a Branson 2210 bath, and cooling to room temperature. Sample vials were rinsed twice with water $(5 \mathrm{~mL} / \mathrm{each})$, and washes were combined with the corresponding samples. After mixing, samples were sonicated for an additional $10 \mathrm{~min}$, centrifuged at $\sim 9,900 \mathrm{~g}$ for $10 \mathrm{~min}$, and 1-mL aliquots of supernatant were filtered through a $0.2-\mu \mathrm{m}$ Gelman Acrodisc ${ }^{\circledR}$ CR PTFE syringe before HPLC analysis (as described above). The percentage of dexamethasone released was calculated as follows:

$\% \mathrm{DEX}=\left(\mathrm{LS}-\mathrm{DEX}_{\mathrm{VH}}\right) /(700 \mu \mathrm{g} \times 100 \%)$, where label strength is the LS and DEX $\mathrm{VH}_{\mathrm{V}}$ is the amount of dexamethasone recovered from the vitreous humor $=$ concentration result from $\mathrm{HPLC} \times$ volume of dissolution solvent $(\mathrm{mL})$.

\section{Determination of dexamethasone concentration in the aqueous and vitreous humors by liquid chromatography-tandem mass spectrometry analysis}

Samples of aqueous humor $(100 \mu \mathrm{L})$ and DEX implant remnant-free vitreous humor $(200 \mu \mathrm{L})$ were mixed with an equal volume of internal standard (beclomethasone $1 \mu \mathrm{g} / \mathrm{mL}$ ) and centrifuged at $\sim 14,000 \mathrm{~g}$ for $12 \mathrm{~min}$. Supernatant was then analyzed by liquid chromatography-tandem mass spectrometry. Separation of dexamethasone and internal standard was achieved with a Waters Xterra $2.5-\mu \mathrm{m}$ column $(50 \times$ $2.1 \mathrm{~mm}$ ) using mobile phase A [0.02\% (w/v) ammonium acetate and $0.04 \%(\mathrm{v} / \mathrm{v})$ acetic acid in water] and phase $\mathrm{B}[0.1 \%(\mathrm{v} / \mathrm{v})$ acetic acid in acetonitrile] according to the following gradient (time in $\mathrm{min} / \% \mathrm{~A}$ ): 0/80, 4/20, 5/20, 6/80, $6 / 80$. Mass spectrometry detection was performed using an API $2000^{\mathrm{TM}}$ triple quadrupole mass spectrometer (AB Sciex, Foster City, CA) equipped with a TurbolonSpray ${ }^{\circledR}$ ionization source. Data acquisition was performed in a positive ion multiple reaction monitoring mode for highly selective detection of the dexamethasone and internal standard in the complex vitreous humor sample matrix. Quantitation was performed based on the internal standard.

\section{Statistical analysis}

The 2-sample $t$-test was used with Minitab ${ }^{\circledR} 15$ (Minitab, Inc., State College, PA) to compare 1-piece and 3-piece DEX implants at individual time points, after exclusion of outliers. Significance was set at $P<0.05$. The extreme studentized deviate $(\mathrm{ESD})^{19}$ procedure was used to detect statistical outliers among samples at a given time point. Because the expected ESD value for a sample size of 6 at a $5 \%$ significance level is $1.82,{ }^{19}$ samples with ESD values greater than 1.82 were marked as outliers and excluded from further analysis.

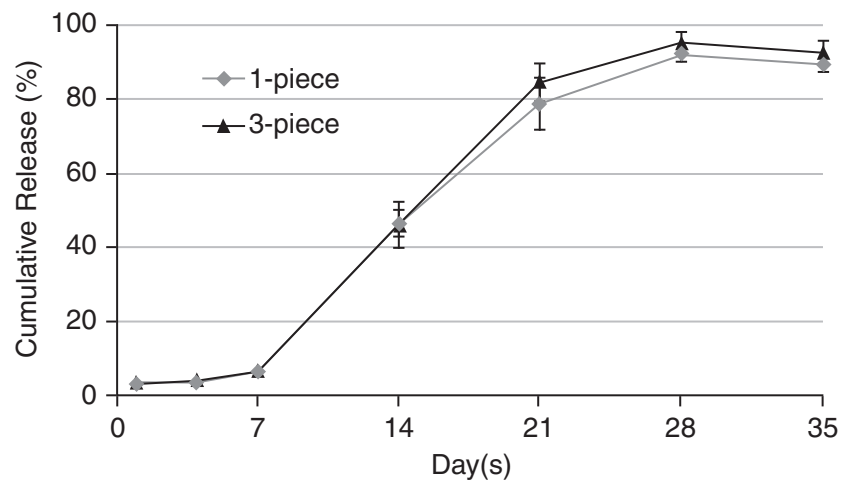

FIG. 1. Cumulative release of dexamethasone from 1piece and 3-piece dexamethasone intravitreal implants (DEX implants) in vitro. Results are expressed as mean percentage \pm standard deviation based on 6 replicates per time point.

\section{Results}

In vitro analyses showed that the 1-piece and 3-piece DEX implants exhibited similar release of dexamethasone over time (Fig. 1); there was no statistically significant difference in vitro between intact and fragmented DEX implants at any of the time points assessed.

In vitro data were supported by in vivo findings. Figure 2 shows that the in vivo release profile of dexamethasone in rabbits was indeed similar to that observed in vitro. A statistically significant difference was noted between the 1-piece and 3-piece DEX implants at day $1(P=0.025)$, although it is expected to have no clinical impact. Otherwise, the release of dexamethasone from 1-piece and 3-piece DEX implants in rabbits was not statistically significant at any other time point $(P \geq 0.167)$, with $>95 \%$ of dexamethasone released by day 28 from both the 1-piece and 3-piece DEX implants (Fig. 2).

In Fig. 3, the concentration profile of dexamethasone indicates that the 1-piece and 3-piece DEX implants exhibited similar pharmacokinetic behavior in the vitreous humor. The concentration of dexamethasone detected after implantation of intact versus fragmented DEX implants in rabbits was not statistically different at any of the time points assessed $(P \geq 0.102)$. In the aqueous humor, a statistically significant

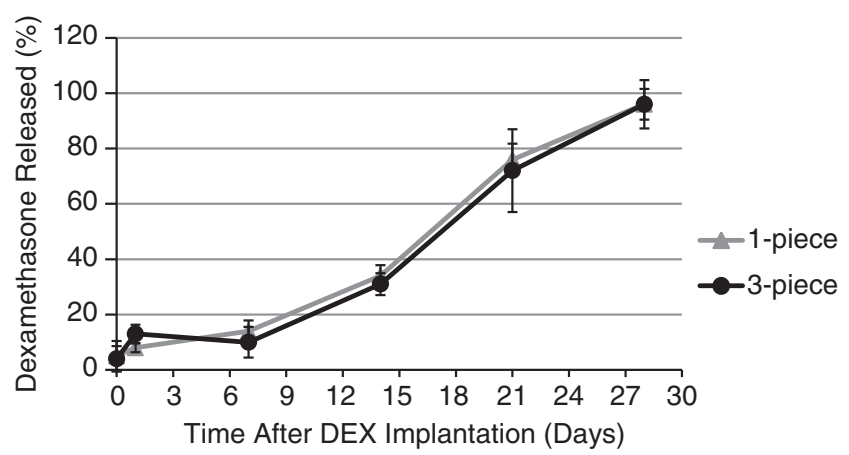

FIG. 2. Cumulative release of dexamethasone in the vitreous humor of rabbits after implantation of 1-piece or 3piece DEX implants in the posterior segment of opposing eyes. Results are expressed as mean percentage \pm standard deviation based on 6 replicates per time point. $P=0.025$ at day 1 , but not significant at any other time point. 


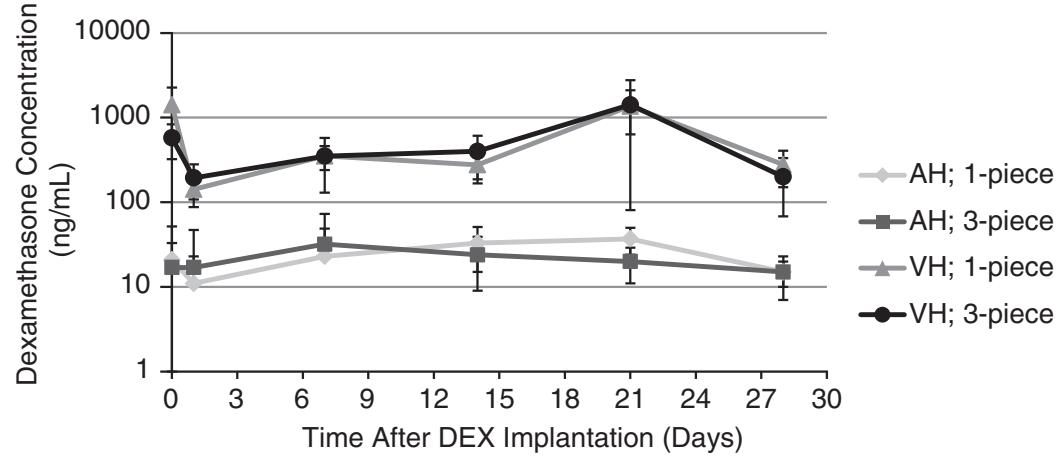

FIG. 3. Dexamethasone concentration in the vitreous and aqueous humors of rabbits after implantation of 1-piece or 3-piece DEX implants in the posterior segment of opposing eyes. Results are expressed as mean concentration $(\mathrm{ng} / \mathrm{mL}) \pm$ standard deviation based on 6 replicates per time point. $P=0.032$ at day 21 in the aqueous humor, but not significant at any other time point in the aqueous humor, and not significant in the vitreous humor. $\mathrm{AH}$, aqueous humor; $\mathrm{VH}$, vitreous humor. difference was observed at day $21(P=0.032)$, although its impact is expected to be negligible (see Discussion section). Otherwise, there was no statistically significant difference at any other time points $(P \geq 0.376)$. Figure 3 also shows that the concentration of dexamethasone stayed constant over time in both the vitreous and aqueous humor. Moreover, the concentration of dexamethasone in the aqueous humor remained minimal over time, hence confirming that the release of dexamethasone was restricted to the vitreous cavity and that the difference between the 1-piece and 3-piece DEX implants observed at day 21 in the aqueous humor is unlikely to be clinically relevant.

\section{Discussion}

The results of this in vivo study in rabbits indicate that fragmentation of DEX implant does not affect its pharmacokinetic behavior. Overall, there was no difference in the percentage of dexamethasone released from 1-piece versus 3-piece DEX implants or in the concentration detected in the corresponding vitreous and aqueous humors over time. These findings are notable because the augmentation in surface area that results from fragmentation could have accelerated dissolution of DEX implant and increased the concentration of dexamethasone delivered at a given time. However, Bourgault and Albiani ${ }^{18}$ determined that following segmentation perpendicular to the long axis of the rodshaped DEX implant (which is the most likely scenario given the $6 \times 0.46 \mathrm{~mm}$ dimensions, and was reported by Roy and Hedge ${ }^{17}$ as well as Rishi et al. ${ }^{16}$ ), the increase in surface area would only amount to $3.7 \%$. The authors thus concluded that a fractured DEX implant was unlikely to have any significant clinical effect, ${ }^{18}$ which is consistent with the data presented herein.

Lee et al. ${ }^{20}$ described the DEX implant drug release process in monkeys and noted that once most of the drug had diffused out of the implant, fragmentation can be observed. Another preclinical study of DEX implant $0.7 \mathrm{mg}$ in monkeys also showed that although the implants eventually fragmented spontaneously when they became smaller and translucent (i.e., from day 60 onward), the fragments continued to degrade slowly without a drug burst. ${ }^{19}$ A high concentration of dexamethasone was detected in the DEX implant remnant-free vitreous humor from day 7 to 60 $\left(C_{\max }=213 \pm 49 \mathrm{ng} / \mathrm{mL}\right)$. However, the concentration decreased rapidly between day 60 and $90,{ }^{21}$ which is inconsistent with the notion that fragmentation of DEX implant increases the release of dexamethasone. The above studies thus suggest that late fragmentation is part of the normal dissolution process of the DEX implant and does not affect the drug release rate.

In the current study, experiments were performed with 3piece DEX implants (even though both case reports of split DEX implants mentioned 2 fragments ${ }^{16,17}$ ) to amplify the effect, if any, on the surface area and subsequent dissolution of the implants. Our results with 3-piece DEX implants revealed no significant difference in the release and concentration profiles, compared with 1-piece DEX implants, thus suggesting that early fragmentation of the DEX implant (as reported Roy and Hedge ${ }^{17}$ and Rishi et al. ${ }^{16}$ ) does not affect the drug release rate either. Importantly, Chang-Lin et al. ${ }^{22}$ previously conducted pharmacokinetic studies of intact DEX implant in rabbits and concluded that dexamethasone remained present in the eyes for at least 31 days (their longest time point), which is consistent with our results showing high levels of dexamethasone for at least 28 days (our longest time point).

Finally, our findings are consistent with the degradation mechanism described by Robinson and Whitcup. ${ }^{3}$ After intravitreal injection of the dry implant, water immediately starts diffusing in the implant pores, causing dexamethasone to diffuse out. As water penetrates deeper into the implant core, the implant swells and the polylactic acid-glycolic acid polymer undergoes a process of random chain scission, which eventually leads to biodegradation and mass loss as the polymer breaks down due to internal cavitation. ${ }^{3}$ Scission/ cracking of the implant over its entire surface is thus an early integral part of the dexamethasone sustained-release mechanism that increases the surface area of the implant and crosssectional fragmentation (as reported by Roy and Hedge ${ }^{17}$ and Rishi et al. ${ }^{16}$ ) is not expected to have an additional significant impact.

These findings thus indicate that fragmentation of DEX implants does not cause faster dissolution compared with intact DEX implant. Consequently, fragmentation of DEX implant does not expose the vitreous humor to higher concentrations of dexamethasone. Anatomic and physiologic differences between rabbit and human eyes should be considered, but the overall results obtained in rabbits and monkeys indicate that fragmentation of DEX implant in itself does not compromise its efficacy or safety.

\section{Acknowledgments}

This study was sponsored by Allergan, Inc. Writing and editorial assistance were provided to the authors by Michele Jacob, PhD, of Evidence Scientific Solutions (Philadelphia, PA) and funded by Allergan, Inc. All authors met the 
ICMJE authorship criteria. Neither honoraria nor payments were made for authorship.

\section{Author Disclosure Statement}

R. Bhagat and X-L. Li are employees of Allergan, Inc. and Jean Zhang and Sidiq Farooq were employees of Oculex at the time of study conduct.

\section{References}

1. Lattanzio, R., Torres Gimeno, A., Battaglia Parodi, M., and Bandello, F. Retinal vein occlusion: current treatment. Ophthalmologica. 225:135-143, 2011.

2. Deobhakta, A., and Chang, L.K. Inflammation in retinal vein occlusion. Int. J. Inflam. 2013:438412, 2013.

3. Robinson, M.R., and Whitcup, S.M. Pharmacologic and clinical profile of dexamethasone intravitreal implant. $E x$ pert Rev. Clin. Pharmacol. 5:629-647, 2012.

4. Hunter, R.S., and Lobo, A.M. Dexamethasone intravitreal implant for the treatment of noninfectious uveitis. Clin. Ophthalmol. 5:1613-1621, 2011.

5. Allergan, Inc. Ozurdex (dexamethasone intravitreal implant) prescribing information. Available at www.allergan .com/assets/pdf/ozurdex_pi.pdf (last accessed April 25, 2014).

6. Herrero-Vanrell, R., Cardillo, J.A., and Kuppermann, B.D. Clinical applications of the sustained-release dexamethasone implant for treatment of macular edema. Clin. Ophthalmol. 5:139-146, 2011.

7. Ferrini, W., and Ambresin, A. Intravitreal dexamethasone implant for the treatment of macular edema after retinal vein occlusion in a clinical setting. Klin. Monbl. Augenheilkd. 230:423-426, 2013.

8. Kuppermann, B.D., Blumenkranz, M.S., Haller, J.A., et al. Randomized controlled study of an intravitreous dexamethasone drug delivery system in patients with persistent macular edema. Arch. Ophthalmol. 125:309-317, 2007.

9. Haller, J.A., Bandello, F., Belfort Jr., R., et al. Randomized, sham-controlled trial of dexamethasone intravitreal implant in patients with macular edema due to retinal vein occlusion. Ophthalmology. 117:1134-1146 e1133, 2010.

10. Lowder, C., Belfort Jr., R., Lightman, S., et al. Dexamethasone intravitreal implant for noninfectious intermediate or posterior uveitis. Arch. Ophthalmol. 129:545-553, 2011.

11. Naik, R.K., Gelhorn, H., Kowalski, J., et al. Efficacy of dexamethasone intravitreal implant for the treatment of uveitis based on patient-reported vision function outcome measures. Invest. Ophthalmol. Vis. Sci. 51:5875, 2010.

12. Myung, J.S., Aaker, G.D., and Kiss, S. Treatment of noninfectious posterior uveitis with dexamethasone intravitreal implant. Clin. Ophthalmol. 4:1423-1426, 2010.

13. Allergan, Inc. Ozurdex (dexamethasone intravitreal implant) 0.7. Available at www.allergan.com/products/eye care/ozurdex.htm. (Last accessed April 25, 2014).

14. United States Securities and Exchange Commission. Annual report pursuant to section13 or 15(d) of the Securities Exchange Act of 1934 (for Allergan, Inc.). Available at www .sec.gov/Archives/edgar/data/850693/000085069312000003/ agn10-k.htm. (Last accessed April 25, 2014).

15. Boyer, D.S., Yoon, Y.H., Belfort Jr., R., et al. Three-year, randomized, sham-controlled trial of dexamethasone intravitreal implant in patients with diabetic macular edema. Ophthalmology 121:1904-1914, 2014.

16. Rishi, P., Mathur, G., and Rishi, E. Fractured Ozurdex implant in the vitreous cavity. Indian J. Ophthalmol. 60:337338, 2012.

17. Roy, R., and Hegde, S. Split Ozurdex implant: a caution. Can. J. Ophthalmol. 48:e15-e16, 2013.

18. Bourgault, S., and Albiani, D. Split Ozurdex implant: a caution. Can. J. Ophthalmol. 48:218-219, 2013.

19. Grubbs, F.E. Procedures for detecting outlying observations in samples. Technometrics. 11:1-21, 1969.

20. Lee, S.S., Hughes, P., Ross, A.D., and Robinson, M.R. Biodegradable implants for sustained drug release in the eye. Pharm. Res. 27:2043-2053, 2010.

21. Chang-Lin, J.E., Attar, M., Acheampong, A.A., et al. Pharmacokinetics and pharmacodynamics of a sustainedrelease dexamethasone intravitreal implant. Invest. Ophthalmol. Vis. Sci. 52:80-86, 2011.

22. Chang-Lin, J.E., Burke, J.A., Peng, Q., et al. Pharmacokinetics of a sustained-release dexamethasone intravitreal implant in vitrectomized and nonvitrectomized eyes. Invest. Ophthalmol. Vis. Sci. 52:4605-4609, 2011.

Received: July 3, 2014 Accepted: October 15, 2014

Address correspondence to: Rahul Bhagat Allergan, Inc. 2525 Dupont Drive Irvine, $C A 92612$

E-mail: bhagat_rahul@allergan.com 